\title{
High-frequency Polarimetric Observations of the Crab Pulsar
}

\author{
A. Karastergiou ${ }^{1,2}$, A. Jessner ${ }^{2} \&$ R. Wielebinski ${ }^{2}$ \\ ${ }^{1}$ School of Physics, University of Sydney, NSW 2006, Australia \\ ${ }^{2}$ Max-Planck Institut für Radioastronomie, Auf dem Hügel 69, 53121 \\ Bonn, Germany
}

\begin{abstract}
We present high quality polarimetric observations with the Effelsberg radio telescope of the Crab pulsar at $8.35 \mathrm{GHz}$ using a new sensitive receiver with a very large bandwidth. We present our observations and discuss them in the context of the conclusions reached by Moffett \& Hankins (1999) about the geometry and the origin of the various pulse components.
\end{abstract}

\section{Understanding the Crab Pulsar}

The Crab pulsar is detectable as a source of pulsed emission from radio to gamma-rays, with the main pulse components in general agreement across the spectrum. The exceptions are the precursor, seen just before the main component at low radio frequencies and the extra components seen at the highest radio frequencies. The position angle (hereafter PA) of these highly polarized components can be used to infer the origin of this radiation in the pulsar magnetosphere and whether it is of the same geometry as the main component and inter-pulse. We have observed the Crab at $8.35 \mathrm{GHz}$ with a new super-sensitive receiver to provide a much-needed high signal-to-noise ratio polarization profile at this frequency and provide an answer to the origin of the high-frequency components (hereafter HF-components).

\section{The Polarization}

Figure 1 shows the profile we obtained at $8.35 \mathrm{GHz}$ over a $1.2 \mathrm{GHz}$ bandwidth. It is immediately notable that both the inter-pulse (the leading, highest intensity component) and the HF-components (the trailing two components) are almost entirely linearly polarized. The PA at each phase bin is plotted directly under the profile, with the scale ranging from $-90^{\circ}$ to $90^{\circ}$. Moffett \& Hankins (1999) attempted to accommodate the PA of the HF-components in the geometry of the main pulse and inter-pulse (the main pulse is not observed at this frequency). Their fit suggested that the angle between the rotation and magnetic axes is $56^{\circ}$, however, the impact parameters for the various components where found to be much larger than the expected polar cap size of the Crab pulsar according to Goldreich \& Julian (1969). Making an unconstrained rotating vector model fit to our data for the inter-pulse and HF-components only, we have obtained angles 


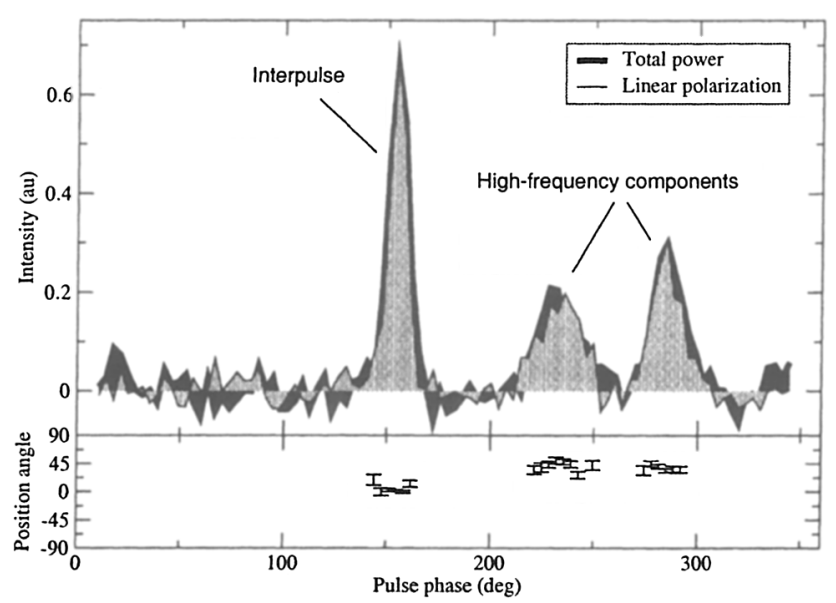

Figure 1. The $8.35 \mathrm{GHz}$ polarization profile of the Crab.

corresponding to a closely aligned rotator (magnetic inclination $\alpha=4^{\circ} \pm 1^{\circ}$, impact parameter $\beta=14_{-8}^{+3} \mathrm{deg}$ ), at variance with previous estimates from the literature. Our fit is however compatible with the usual phase angle of the main pulse and the low-frequency precursor, but only planned $5 \mathrm{GHz}$ observations will enable us to give a more precise result. Observations in the optical (Narayan \& Vivekanand 1982) and X-rays (Weisskopf et al. 2000) can also be instrumental in constraining the geometry.

\section{Our First Conclusions}

The high quality data obtained with the Effelsberg telescope show a very flat position angle and no significant position angle variation between the two HFcomponents. If they do form a conal pair, as suggested by Moffett \& Hankins (1999), the lack of swing could be interpreted as a full 180 degree swing of the position angle. The polarization position angle of the high-frequency components cannot be accommodated by the geometry of the other components without major discrepancies. In trying to fit the rotating vector model, it is necessary to consider the effects of retardation, which will induce delays due to the difference in emission height of the different components. At present, we are working with data from all frequencies observed to produce the most plausible solution for the origin of the various components and the orientation of the neutron star by trying to match different magnetospheric models.

\section{References}

Goldreich, P., \& Julian, W. H. 1969, ApJ, 157, 869

Moffett, D. A., \& Hankins, T. H. 1999, ApJ, 522, 1046

Narayan, R., \& Vivekanand, M. 1982, A\&A, 113., L3

Weisskopf, M. C., et al. 2000, ApJ, 536, L81 